\title{
An optimised perfusion technique for extracting murine gastric leukocytes
}

\author{
Garrett $\mathrm{Z} \mathrm{Ng}^{1 \#}$ and Philip Sutton ${ }^{1,2}$
}

${ }^{1}$ Mucosal Immunology, Murdoch Childrens Research Institute, Royal Children's Hospital, Parkville, Victoria, Australia

${ }^{2}$ Centre for Animal Biotechnology, School of Veterinary and Agricultural Science, University of Melbourne, Parkville, Victoria, Australia

${ }^{\#}$ To whom correspondence should be addressed.

Dr Garrett Z Ng,

Mucosal Immunology, Murdoch Childrens Research Institute, Royal Children's Hospital, Melbourne, VIC, Australia

garrett.ng@mcri.edu.au; Ph: +61-3-9936-6624; Fax: +61-3-9936-6528

Keywords: stomach, leukocytes, flow cytometry

Format: Technical note 


\section{ABSTRACT}

The stomach is a difficult tissue to analyse by flow cytometry, largely due to the difficulty of isolating viable leukocytes. Here we present the re-optimization of a perfusion technique that compares favourably against two other methods of enzymatic digestion for the release of gastric leukocytes. We believe this technique could greatly assist analysis of immune cells basally present in the murine stomach and that infiltrate during infection or disease.

\section{INTRODUCTION}

While leukocyte isolation from the intestinal compartment of mice is relatively well established [1], only a limited number have focused on the gastric compartment [2-4]. Indeed, immune cells in the stomach are generally considered as notoriously difficult to analyse by flow cytometry. This limitation has contributed to our poor understanding of the immunology of the stomach. Improved methods of isolating leukocytes would greatly assist studies aimed at examining gastric immunology.

As the stomach is a quite fibrous tissue, most descriptions use enzymatic digestion (primarily collagenases) to successfully dissociate gastric cells into a suspension. There are however drawbacks to using enzymatic digestion to release cells from tissues. Often collagenases can have a high degree of lot-to-lot variability which results in differential activity [5]. Excessive enzymatic digestion may reduce cell viability, or degrade cellular antigens $[1,6]$, resulting in variation in antibody labelling.

Here we describe the optimization of a reliable and reproducible method for the isolation of gastric leukocytes for flow cytometric analysis that does not require digestion of stomach tissue. This method is compared to alternative enzymatic methods for isolating gastric leukocytes. 


\section{MATERIALS AND METHODS}

\section{Perfusion}

Gastric cellular infiltrates to be analysed by flow cytometry were isolated from matched 8-12 week old specific-pathogen free C57BL/6 and NOD-scid $I l 2 \mathrm{rg}^{-/}$(NSG) mice [7] by perfusion using a technique modified from Alderuccio et al [2]. Stomachs, opened along the inner curvature and contents discarded, were collected into HBSS (Gibco) then perfused with $\sim 7 \mathrm{~mL}$ of HBSS containing 5\% FCS (Gibco), 5 $\mathrm{mM}$ EDTA and/or $1 \mathrm{mM}$ dithiothreitol (perfusion solution). This was performed by slowly inserting a $25 \mathrm{G}$ needle at a very shallow angle into the mucosa near the limiting ridge, while expelling perfusion solution. This caused the mucosa to swell, and further fluid was then injected into sites adjacent to the swelling to expand the disrupted locations. This was repeated as necessary until all mucosa was fully inflated. Perfused stomachs were incubated at $37^{\circ} \mathrm{C}$ for $15 \mathrm{~min}$ then cut into $\sim 0.5 \mathrm{~cm}$ pieces and poured through a $70 \mu \mathrm{M}$ cell strainer. After a further $10 \mathrm{~min}$ in $10 \mathrm{~mL}$ of fresh perfusion solution, pieces were vortexed and again poured through a $70 \mu \mathrm{M}$ cell strainer. Cells in both filtrates were pooled and collected by centrifugation at $600 \mathrm{~g}$ for $5 \mathrm{~min}$.

\section{Enzymatic digestion of stomachs}

Stomachs were digested with collagenase as previously described [8]. Briefly, stomachs were cut into $\sim 0.5 \mathrm{~mm}$ pieces then centrifuged at $150 \mathrm{~g}$ for $5 \mathrm{~min}$. The pellet was resuspended in $7 \mathrm{~mL}$ of HBSS containing $0.2 \%$ BSA and $0.4 \mathrm{mg} / \mathrm{mL}$ collagenase A (Roche) and incubated shaking at $37^{\circ} \mathrm{C}, 120 \mathrm{rpm}$. Ten $\mathrm{mL}$ complete DMEM was added and digested tissue dissociated by three repeats of forceful pipetting using serological pipettes for 5 mins. Alternatively the Lamina Propria Kit and the gentleMACS dissociator (Miltenyi Biotec) was used as per manufacturer's instructions. Cell suspensions were filtered through a $70 \mu \mathrm{M}$ cell strainer.

\section{Flow cytometry}

Cells were blocked in $100 \mu \mathrm{L}$ of $20 \%$ normal mouse serum (collected in house) and $1 \mu \mathrm{g} / \mathrm{mL}$ anti-Fc $\gamma \mathrm{II} / \mathrm{Fc} \gamma \mathrm{III}$ (2.4G2, Walter and Eliza Hall Institute) on ice for 20 min. Cells were stained with anti-CD45-PE.Cy5.5 or anti-CD45-Alexa 700 (30-F11), anti-CD11c-FITC (M5/114.15.2; eBioscience, San Diego, CA, USA), anti-CD11bBV421 (M1/70), anti-CD19-BV510 (6D5), anti-CD4-BV650 (RM4-5), anti-CD103- 
BV786 (2E7), anti-CD64-PE (X54-5/7.1), anti-MHCII-PE.Cy7 (M5/114.15.2) and anti-Ly6G-APC.Cy7 (1A8; all from BioLegend, San Diego, CA, USA unless indicated). Cells were washed twice, resuspended in $0.25 \mathrm{mg} / \mathrm{mL}$ propidium iodide (ImmunoChemistry Technologies, Bloomington, MN, USA) and acquired on a BD LSRFortessa X-20 flow cytometer (BD Biosciences, San Jose, CA, USA). Single cells were identified as a linear population on FSC-A vs FSC-H then propidium iodide positive dead cells excluded. Cell counts were normalised using AccuCount Fluorescent Particles (Spherotech, Lake Forest, IL, USA). Data were analysed using FCS Express (De Novo Software, Los Angeles, CA, USA).

\section{Statistics}

Data with multiple comparison groups was analysed by two-way ANOVA (GraphPad Prism 5.0, GraphPad Software, San Diego, CA, USA), or one-way ANOVA with Dunnett's post hoc test (SPSS statistics 21, IBM, Armonk, NY, USA).

\section{RESULTS}

\section{Optimization of the perfusion technique}

It was originally described to use PBS to perfuse mouse stomachs [2]. We have anecdotally noticed in unrelated studies that gastric stromal cells have higher viability when kept in HBSS. Additionally, we wondered if a higher yield of cells could be obtained if EDTA or DTT were added to the perfusion buffer, as these chemicals are well known to promote the release of cells from tissue and dissolve mucus respectively. When we tested HBSS with or without the addition of EDTA and/or DTT, both significantly increased the yield of CD45+ cells, with the highest numbers released from using both EDTA and DTT (Figure 1A).

We wondered if an additional incubation(s) in EDTA/DTT would result in higher cell yields. Stomachs were perfused with buffer containing EDTA/DTT then incubated for 15 mins followed by further $10 \mathrm{~min}$ incubation(s). Maximum leukocyte isolation was achieved following an initial $15 \mathrm{~min}$ incubation followed by one $10 \mathrm{~min}$ incubation in fresh buffer (Figure 1C). No further improvement was observed by addition of a second incubation step.

We also tested whether more cells could be isolated by further mechanical processing. This involved separating the mucosa from the muscularis mucosae by either scraping off the layer using a scalpel blade (as originally recommended [2]) or 
by processing the tissue through a $70 \mu \mathrm{m}$ strainer. Unexpectedly, both of these treatments significantly reduced the number of CD45+ cells (Figure 1B). Visible clumping of the cell suspensions was also observed, which we believe could be due to cell damage caused by the extra mechanical treatments.

From these experiments we considered perfusion with HBSS containing $5 \mathrm{mM}$ EDTA and $1 \mathrm{mM}$ DTT followed by a $15 \mathrm{~min}$ incubation, then placing the stomachs in fresh buffer for a further 10 mins to be optimal for leukocyte extraction.

\section{Comparison of different methods to isolate murine gastric leukocytes}

We compared our optimised perfusion technique with two standard digestion techniques, namely digestion with collagenase A as we performed previously for the isolation of gastric epithelial cells [9] and dissociation with a Lamina Propria Dissociation Kit and the gentleMACS dissociator (Miltenyi Biotec). The number of CD45+ cells that we obtained following perfusion was significantly higher than with either of these methods (Figure 1D). As above, visible aggregation of the cell suspension could also be observed which suggested viability was affected with both enzymatic digestions.

\section{Analysis of gastric leukocytes in C57BL/6 and NSG mice}

We applied the perfusion technique to analyse the cells present in the stomachs of naïve C57BL/6 and NSG mice, which have severe deficiency of T cells, B cells, NK cells, as well as further defects in macrophages and dendritic cells [7]. Using the gating strategy shown in Figure 2A, we were able to identify and detect differences in multiple populations of leukocytes between strains of mouse. To demonstrate the specificity of these analyses, NSG mice were used as negative controls; as expected, cells isolated from these mice by the optimised perfusion technique, lacked most other leukocyte subsets except for neutrophils (Figure 2B).

\section{DISCUSSION}

Here we present the optimization and comparison of a perfusion technique for isolating gastric leukocytes for flow cytometric analysis. The advantages of this optimised technique are that it requires less time than enzymatic digestion, that degradation of antigenic epitopes is not a potential issue and the increased yield of 
leukocytes obtained. It is also likely that intraepithelial lymphocytes are also obtained, a population unlikely to be represented in the original perfusion description.

The stomach is more fibrous than the lower GI tract, which might explain why it is more difficult to successfully digest than intestine. We believe a clue to the poor performance of enzymatic digestion may be the obvious aggregation of cell suspensions obtained. Damaged or dying cells can release 'sticky' genomic DNA, which might cause the aggregation of cells in suspension. These cells are then lost, either from necessary filtration steps prior to flow cytometric analysis or excluded from analysis as aggregates.

Although convincing flow cytometric analysis of leukocytes during $H$. pylori infection has been performed [4], isolation of sufficient numbers of cells to phenotype gastric leukocytes from individual naïve mice is difficult. Therefore, this technique provides a significant technical improvement. From the data presented it is clear that minor populations of leukocytes present in the naïve stomach can be detected and analysed. Further, we have used this technique to isolate approximately 300-500 gastric macrophages by FACS based on 5 marker analysis from individual naïve mice for gene expression analysis by quantitative PCR [10]. These approaches to analysing subsets of leukocytes from the naïve mouse stomach could greatly aid understanding the immunology of the stomach.

\section{ACKNOWLEDGEMENTS}

The authors thank Matthew Burton for technical assistance. This study was supported by the Victorian Government's Operational Infrastructure Support Program and by Project Grant GNT1046254 from the National Health and Medical Research Council of Australia. PS is supported by a Senior Research Fellowship from the NHMRC. 


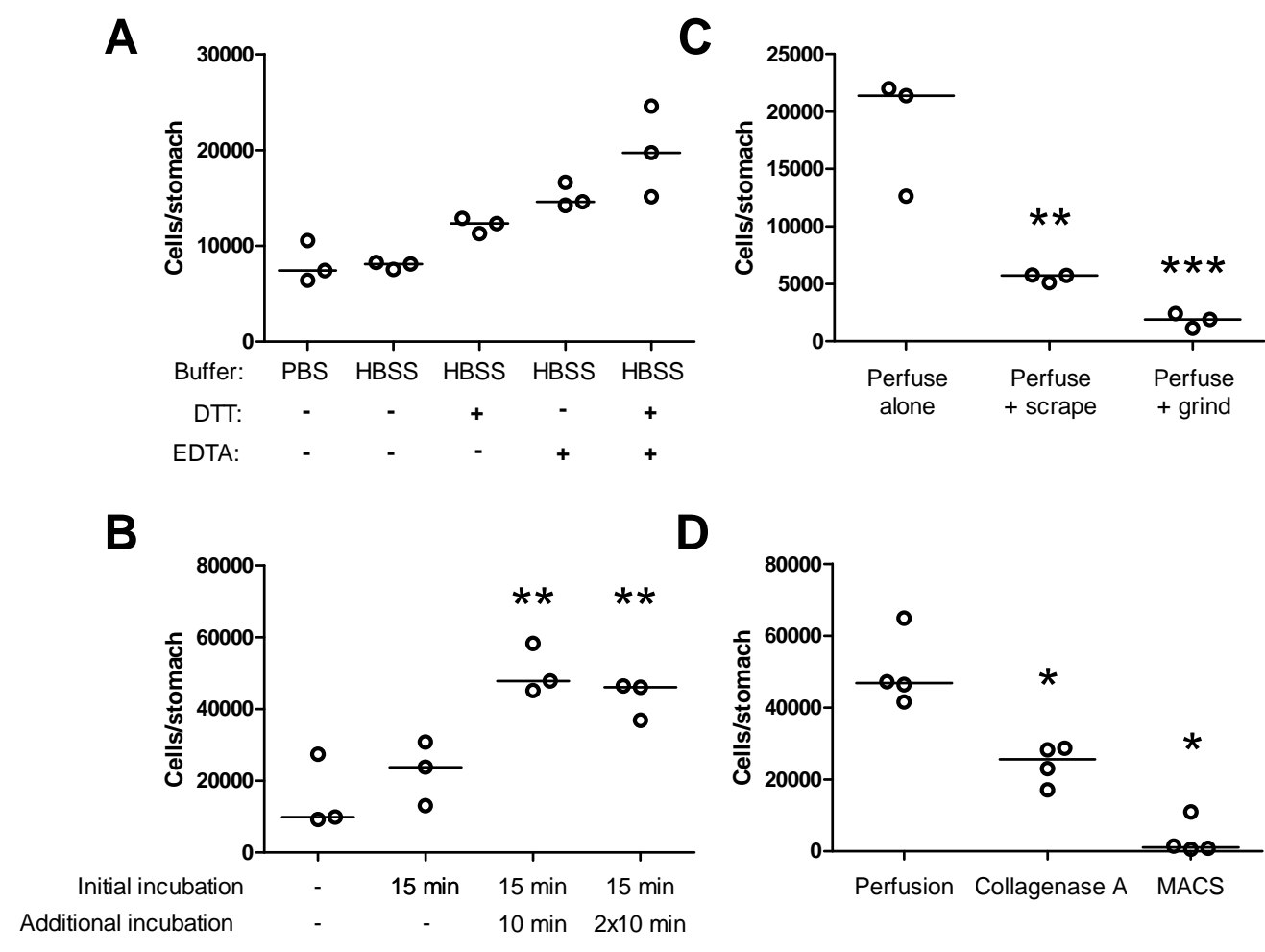

Figure 1: Optimization of the perfusion technique and comparison to enzymatic digestion. (A) Stomachs were perfused with PBS or HBSS with $1 \mathrm{mM}$ DTT and/or 5 mM EDTA and the cells isolated analysed by flow cytometry to quantify CD45+ cells. *Both DTT and EDTA significantly increased the number of CD45+ cells ( $p=0.01$ and $p=0.0009$ respectively; Two-way ANOVA) compared to controls. (B) Stomachs were perfused with HBSS plus DTT/EDTA then the mucosa separated by scraping with a scalpel blade or the stomach ground through a $70 \mu \mathrm{M}$ strainer. Both mechanical treatments significantly reduced CD45+ cell yield (ANOVA cf perfusion alone). (C) Stomachs were perfused with HBSS plus DTT/EDTA and then incubated at $37^{\circ} \mathrm{C}$ for $15 \mathrm{~min}$ followed by further incubations for $10 \mathrm{~min}$ (ANOVA cf no incubation). (D) $\mathrm{CD} 45+$ cells isolated with the final perfusion procedure was compared to the efficacy of Collagenase A digest and dissociation using the Lamina Propria Kit and gentleMACS dissociator (Miltenyi Biotec). Perfusion resulted in the isolation of significantly more CD45+ cells (ANOVA cf perfusion alone). All experiments used stomachs from C57BL/6 mice. Graphs present individual stomachs (points) and median (bar); ${ }^{*} \mathrm{p}<0.05, * * \mathrm{p}<0.01,{ }^{*} * \mathrm{p}<0.001$ (ANOVA). 

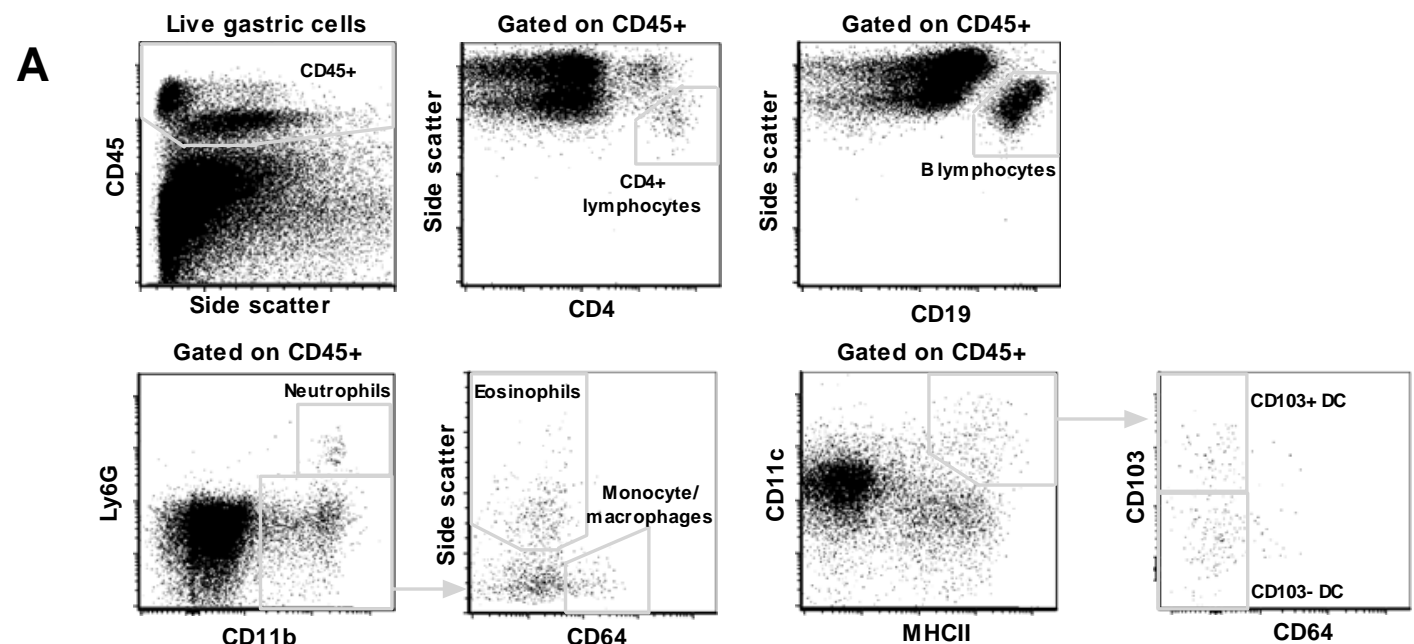

B
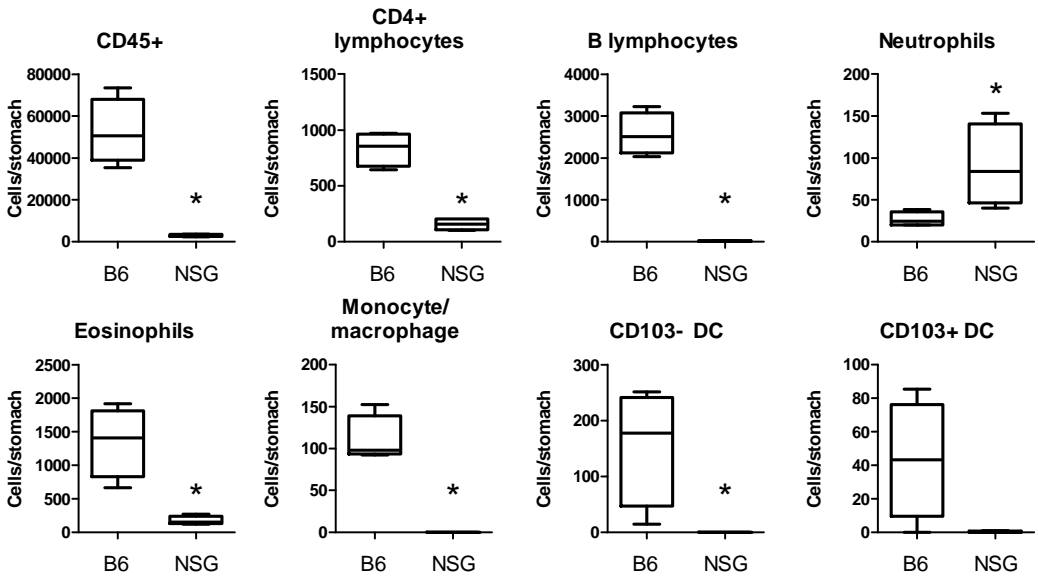

Figure 2: Flow cytometric analysis of gastric leukocytes from C57BL/6 and NSG mice. Leukocytes isolated from the stomachs of naïve C57BL/6 and NSG mice $(n=4)$ using the optimised perfusion protocol were analysed by flow cytometry. (A) Representative gating strategy used to identify subsets of leukocytes. (B) Numbers of each subset in individual mice (). Graphs present the median (horizontal bar), interquartile range (box) and $10^{\text {th }}$ and $90^{\text {th }}$ percentiles (bars). 


\section{REFERENCES}

1. Goodyear, A.W., et al., Optimization of murine small intestine leukocyte isolation for global immune phenotype analysis. J Immunol Methods, 2014. 405(0): p. 97-108.

2. Alderuccio, F., et al., A novel method for isolating mononuclear cells from the stomachs of mice with experimental autoimmune gastritis. Autoimmunity, 1995. 21(3): p. 215-221.

3. Ruiz, V.E., et al., Isolating, immunophenotyping and ex vivo stimulation of $\mathrm{CD} 4+$ and $\mathrm{CD} 8+$ gastric lymphocytes during murine Helicobacter pylori infection. J Immunol Methods, 2012. 384(1-2): p. 157-163.

4. Quiding-Järbrink, M., S. Raghavan, and M. Sundquist, Enhanced M1 macrophage polarization in human Helicobacter pylori-associated atrophic gastritis and in vaccinated mice. PLoS One, 2010. 5(11): p. e15018.

5. Van der Heijden, P.J. and W. Stok, Improved procedure for the isolation of functionally active lymphoid cells from the murine intestine. J Immunol Methods, 1987. 103(2): p. 161-167.

6. Van Damme, N., et al., Chemical agents and enzymes used for the extraction of gut lymphocytes influence flow cytometric detection of $T$ cell surface markers. J Immunol Methods, 2000. 236(1-2): p. 27-35.

7. Ito, M., et al., NOD/SCID/ $\gamma$ formula mouse: an excellent recipient mouse model for engraftment of human cells. Blood, 2002. 100(9): p. 3175-3182.

8. Viala, J., et al., Nod1 responds to peptidoglycan delivered by the Helicobacter pylori cag pathogenicity island. Nat Immunol, 2004. 5(11): p. 1166-1174.

9. McGuckin, M.A., et al., Mucl mucin limits both Helicobacter pylori colonization of the murine gastric mucosa and associated gastritis. Gastroenterology, 2007. 133(4): p. 1210-1218.

10. Chionh, Y.T., et al., Protease-activated receptor 1 suppresses Helicobacter pylori gastritis via the inhibition of macrophage cytokine secretion and interferon regulatory factor 5. Mucosal Immunol, 2015. 8(1): p. 68-79. 